\title{
Lactobacillus Regulates Caenorhabditis elegans Cell Signaling to Combat Salmonella Infection
}

\begin{abstract}
Mengzhou Zhou 1,2, Xiaozhen Liu ${ }^{2,3}$, Hai Yu² and Joshua Gong ${ }^{2 *}$
"National "111" Center for Cellular Regulation and Molecular Pharmaceutics, Key Laboratory of Fermentation Engineering (Ministry of Education), School of Food and Biological Engineering, Hubei University of Technology, Hubei, China, ${ }^{2}$ Guelph Research and Development Centre, Agriculture and Agri-Food Canada, Guelph, ON, Canada, ${ }^{3}$ Engineering Research Center of Health Food Design \& Nutrition Regulation, School of Chemical Engineering and Energy Technology, Dongguan University of Technology, Dongguan, China
\end{abstract}

Salmonella typhimurium DT104 infection causes the death of Caenorhabditis elegans, which can be prevented by certain Lactobacillus isolates. However, the molecular mechanisms of both the host response to the infection and the protection by Lactobacillus are largely unclear. The present study has investigated the life-span and gene expression of both wild-type (WT) and mutants in some key components of cell

OPEN ACCESS

Edited by:

Qixiao Zhai,

Jiangnan University, China

Reviewed by:

Julang Li,

University of Guelph, Canada

Bailiang Li,

Northeast Agricultural

University, China

*Correspondence:

Joshua Gong

joshua.gong@canada.ca

Specialty section:

This article was submitted to

Microbial Immunology,

a section of the journal

Frontiers in Immunology

Received: 14 January 2021 Accepted: 12 February 2021

Published: 08 March 2021

Citation:

Zhou M, Liu X, Yu H and Gong J (2021) Lactobacillus Regulates Caenorhabditis elegans Cell Signaling to Combat Salmonella Infection.

Front. Immunol. 12:653205. doi: 10.3389/fimmu.2021.653205 signaling in response to $S$. typhimurium infection and protection from Lactobacillus zeae. The results indicated that the gene expression of daf-16 in the DAF/ insulin-like growth factor (DAF/IGF) pathway, ced-3 and ced-9 in the programmed cell death (PCD) pathway, lys-7, spp-1, and abf-3 for antimicrobial peptide production, and bar-1 involved in the production of other defense molecules was all significantly upregulated when the wild-type (WT) was subjected to DT104 infection. On the contrary, the gene expression of tir-1, sek-1, and pmk-1 in the p38 mitogen-activated protein kinase (MAPK) pathway and clec-60, sod-3, and skn-1 for the production of other defense molecules was significantly suppressed by DT104. Pretreatment of the worms with L. zeae LB1 significantly upregulated the expression of almost all the tested genes except for ced-3, ced-9, abf-2, age-1, and dbl-1 compared with the nematode infected with DT104 only. Mutants defective in the cell signaling or other defense molecules of $C$. elegans were either more susceptible (defective in nsy-1, sek-1, pmk-1, ced-3, ced-9, skn-1, or daf-16) or more resistant (defective in age-1 or dbl-1) to DT104 infection than the WT except for the mutant defective in sod-3. Mutants defective in antimicrobial peptides (lys-7 or abf3) were also more susceptible than the WT. In contrast, the mutant defective in spp-1 became more resistant. When all the mutants were pretreated with $L$. zeae LB1, five mutants that are defective in nsy-1, sek-1, pmk-1, abf-3, or lys-7 showed no response to the protection from LB1. These results suggest that L. zeae LB1 can regulate $C$. elegans cell signaling including the p38 MAPK pathway and downstream production of antimicrobial peptides and defense molecules to combat Salmonella infection.

Keywords: Lactobacillus, Salmonella, Caenorhabditis elegans, cell signaling, antimicrobial peptides, defense molecules 


\section{INTRODUCTION}

Probiotics have long been used to improve animal/human health. Antagonizing enteric infections and enhancing host immune responses are among the crucial benefits of probiotics $(1,2)$. Probiotics are normally commensal bacteria in the mammalian gastrointestinal tract. Probiotics and their products have important roles in gut homeostasis and the functions of innate and adaptive immune systems $(3,4)$. Salmonella is a wellknown enteric pathogen that causes human food- and waterborne illnesses and even death. Development of effective control strategies and technologies, including the use of probiotics, has been extensively studied in the past (5-7). A highlight from these studies is the application of Caenorhabditis elegans as an animal model for efficient selection of probiotic candidates (8-10).

Caenorhabditis elegans is a small soil nematode that can consume bacteria as its food. It has been used extensively as an experimental model to study bacterium and host interactions due to its short reproductive life cycle, clear genetic background, ease of culturing and genetic manipulation, and availability of mutants (11-13). Although the nematode lacks many defense mechanisms presented in higher organisms, it still has complexity and specificity responding to different bacteria at the level of immune regulation, innate immunity in particular, which makes it suitable for elucidating the molecular mechanisms between bacterium and host interactions (14). Previous studies have shown that the worms can be infected and killed by different bacterial pathogens, including Pseudomonas aeruginosa (11), Salmonella enterica $(15,16)$, Staphylococcus aureus (17), Enterococcus faecalis (12), and enterotoxigenic Escherichia coli (ETEC; 18). In addition, a broad overlap of the bacterial virulence factors required for pathogenesis has been found between mammals and C. elegans $(12,18)$. Thus, C. elegans has increasingly been used to help screen the efficacy of probiotics for pathogen control $(8,9,19,20)$. By using C. elegans, we have previously identified several Lactobacillus isolates with the ability to antagonize Salmonella infection to the nematode (9). The protection offered by one of the isolates, named Lactobacillus zeae LB1, has been found to be mediated by a neurotransmitter dopamine through regulation of cell signaling in C. elegans (21). Furthermore, this isolate has demonstrated the ability to attenuate Salmonella infection in the spleen and liver of broiler chickens and reduce Salmonella SPI-1 virulence gene expression in the chicken cecum (22). However, the molecular mechanisms underlying the protective effects, including the regulation of cell signaling, remain to be further elucidated.

It is known that C. elegans immune defense mechanisms are evolutionarily conserved, including the DAF/insulin-like growth factor (DAF/IGF) pathway, p38 mitogen-activated protein kinase (p38 MAPK) pathway, the transforming growth factor- $\beta$ (TGF$\beta$ ) signaling pathway and the programmed cell death (PCD) pathway (23-27). The C. elegans innate immune response consists of the production of numerous antimicrobial proteins, many of which are produced from genes that are induced upon pathogen infection (28-30). Moreover, the expression of different putative antimicrobials involved in the defense of both nematodes and mammals against infection by different pathogens can be regulated by signaling pathways $(28,29$, 31). By investigating the life-span of $C$. elegans and the corresponding gene expression of key components in its cell signaling and defense pathways, including the production of antimicrobial peptides and other defense molecules when exposed to Salmonella typhimurium DT104 and Lactobacillus, this study has determined that L. zeae LB1 regulates C. elegans cell signaling pathways to combat Salmonella infection. The results are reported herein.

\section{MATERIALS AND METHODS}

\section{Caenorhabditis elegans and Bacteria}

Caenorhabditis elegans N2 Bristol wild-type and mutants that are defective in lys-7 (mutant ok1384), nsy-1 (mutant ag3), pmk-1 (mutant km25), sek-1 (mutant ag1), skn-1 (mutant zu67), dbl1 (mutant nk3), spp-1 (mutant ok2703), abf-3 (mutant ok3366), daf-16 (mutant mu86), age-1 (mutant hx546), sod-3 (mutant gk235), ced-9 (mutant n1950), or ced-3 (mutant n717), and the double mutant ced-9;ced-3 (mutant n2812/n717) were obtained from Caenorhabditis Genetics Center (CGC), University of Minnesota, Minnepolis, USA. Caenorhabditis elegans strains were routinely maintained on nematode growth medium (NGM) plates seeded with E. coli OP50 using standard procedures (32).

S. typhimurium DT104 is a porcine multiantibiotic-resistant isolate (33). This strain was cultured on tryptic soy broth (TSB) or tryptic soy agar at $37^{\circ} \mathrm{C}$ for $16 \mathrm{~h}$. Following three washes with M9 medium, $200 \mu \mathrm{l}$ of cell suspension $\left(10^{8} \mathrm{CFU} / \mathrm{ml}\right)$ was spread on a NGM plate ( $100 \mathrm{~mm}$ in diameter) and dried for $3 \mathrm{~h}$ at $22^{\circ} \mathrm{C}$ before beginning of the life-span assay. L. zeae LB1 was grown in de Man Rogosa Sharpe (MRS) broth or on MRS agar at $37^{\circ} \mathrm{C}$ for $18-24 \mathrm{~h}$ in an anaerobic chamber (Coy Laboratory Products, Grass Lake, MI) with an atmosphere of $85 \% \mathrm{~N}_{2}, 10 \% \mathrm{CO}_{2}$, and $5 \% \mathrm{H}_{2}$ (9). After three washes with M9 medium, $200 \mu \mathrm{l}$ cell suspension of $L$. zeae LB1 $\left(10^{8} \mathrm{CFU} / \mathrm{ml}\right)$ was spread on a NGM plate $(100 \mathrm{~mm}$ in diameter) and dried for $3 \mathrm{~h}$ at $22^{\circ} \mathrm{C}$ prior to the use.

\section{Life-Span Assay of C. elegans}

The life-span assays of $C$. elegans were performed using the published methods with some modifications $(8,19,34)$. Briefly, the synchronized C. elegans were transferred to NGM agar with E. coli OP50 and incubated at $25^{\circ} \mathrm{C}$ for $48-60 \mathrm{~h}$ until they reached the L4 stage. In the assays to evaluate the protective effect of $L$. zeae LB1 on nematodes, 50 of L4 stage worms were transferred onto the agar plates seeded with either E. coli OP50 or LB1 followed by incubation at $25^{\circ} \mathrm{C}$, which was designated as day 0 . After $18 \mathrm{~h}$ incubation worms on each plate were transferred to a fresh NGM plate daily that were seeded with DT104 and incubated at $25^{\circ} \mathrm{C}$. In parallel, worms within the control group were transferred to a fresh NGM plate daily that had been seeded with E. coli OP50 after the $18 \mathrm{~h}$ incubation with the same bacterium. The survival of nematode was examined at $24-\mathrm{h}$ intervals up to 15 days. To determine the survival of C. elegans, the number of live worms was recorded daily, and the percentage of surviving worms was calculated by the following formula: survival $(\%)=($ live worms/total worms used $) \times 100$. A worm was considered to be dead when it failed to respond to touch. In 
assays where a mutant was examined, the procedure remained unchanged. Each assay was repeated at least twice unless it is otherwise indicated.

\section{RNA Extraction}

Approximately 150 worms were sampled from each treatment on day 2 of the life-span assays and were then subjected to RNA extraction. The nematodes were washed and disrupted using the method described previously (34) before RNA extraction with the mirVana miRNA Isolation Kit. The RNA integrity was determined by visualization in an agarose gel after treating with DNase I (Ambion, TX) followed by verified as DNAfree by PCR assays. The RNA concentration was determined with a NanoDrop ND-1000 spectrophotometer (NanoDrop Technologies, Wilmington, DE, USA).

\section{Reverse Transcription and Quantitative PCR Analysis}

Caenorhabditis elegans gene expression was determined by quantitative PCR (QPCR) analysis after reverse transcription using SuperScript first-strand synthesis system (Invitrogen, Carlsbad, CA, USA). Two housekeeping genes Gapdh and Act1 were used as internal controls. QPCR assays were performed using 7,500 Real Time PCR System (Applied Biosystems, Foster, CA, USA) and brilliant SYBR green QPCR master mix (Bio-Rad Laboratories, Richmond, VA) following the program: $5 \mathrm{~min}$ at $95^{\circ} \mathrm{C}$ and 40 cycles of $95^{\circ} \mathrm{C}$ for $30 \mathrm{~s}, 56^{\circ} \mathrm{C}$ for $1 \mathrm{~min}$, and $72^{\circ} \mathrm{C}$ for 30 s. For QPCR assays, each tube contained $12.5 \mu$ l Master Mix, $3.75 \mu \mathrm{l}$ each of the primers at $150 \mathrm{nM}, 1 \mu \mathrm{l} \mathrm{cDNA}$ sample, and 4 $\mu \mathrm{l}$ irradiated and double autoclaved $\mathrm{dH} 2 \mathrm{O}$. The PCR primers are listed in Table 1.

The target gene expression was calculated using the $2^{-\Delta \Delta \mathrm{Ct}}$ method (35). The $\Delta \mathrm{Ct}$ represents the difference between the $\mathrm{Ct}$ value with the primers to a target gene and the $\mathrm{Ct}$ value to the housekeeping genes. The $\Delta \Delta \mathrm{Ct}$ represents the difference between the $\Delta \mathrm{Ct}$ value of treatment group (either treated with Lactobacillus or Salmonella) and the $\Delta \mathrm{Ct}$ value of control group (treated with E. coli OP50). The values derived from $2^{-\Delta \Delta \mathrm{Ct}}$ represent fold changes of samples in abundance relative to the reference samples. The reference samples (treated with E. coli OP50) had the $2^{-\Delta \Delta \mathrm{Ct}}$ value of 1 .

\section{RESULTS}

\section{Enhancement in the Resistance of $C$. elegans to DT104 Infection by LB1}

Figure 1 shows the effect of isolate LB1 on the life-span of C. elegans infected with S. typhimurium DT104. Although pretreatment of the WT nematode (N2) with isolate L. zeae LB1 did not eliminate death caused by DT104, LB1 pretreatment significantly extended $(P \leq 0.05)$ the life-span of the worms infected with DT104 only. These results were similar to our previously reported observations with a temperature-sensitive mutant (SS104) of C. elegans for preselection of probiotic candidates (9).
TABLE 1 | Primers of QPCR assay .

\begin{tabular}{|c|c|c|c|}
\hline Primer & Amplicon (bp) & Sequence $\left(5^{\prime}\right.$ to $\left.3^{\prime}\right)$ & $\begin{array}{l}\text { Source or } \\
\text { Reference }\end{array}$ \\
\hline Act-1-F & 121 & CCССACTCAATCCAAAGGCT & (34) \\
\hline Act-1-R & & GTACGTCCGGAAGCGTAGAG & \\
\hline Daf-16-F & 181 & TCGTCTCGTGTTTCTCCAGC & (34) \\
\hline Daf-16-R & & TAATCGGCTTCGACTCCTGC & \\
\hline Age-1-F & 359 & СТCCTGAACCGACTGCCAAT & (34) \\
\hline Age-1-R & & AAATGCGAGTTCGGAGAGCA & \\
\hline Lys-7-F & 153 & GTACAGCGGTGGAGTCACTG & (34) \\
\hline Lys-7-R & & GCCTTGAGCACATTTCCAGC & \\
\hline Clec-60-F & 219 & CGGTITCAATGCGGTATGGC & (34) \\
\hline Clec-60-R & & TGAAGCTGTGGTTGAGGCAT & \\
\hline Clec-85-F & 121 & CCAATGGGATGACGGAACCA & (34) \\
\hline Clec-85-R & & СTTCTGTCCAGCCAACGTCT & \\
\hline Abf-3-F & 189 & AACAGATTGGGGTCAGCTCG & (34) \\
\hline Abf-3-R & & TGGAGACCATTATTGCCGGG & \\
\hline Spp-1-F & 106 & TGGACTATGCTGTTGCCGTT & (34) \\
\hline Spp-1-R & & ACGCCTTGTCTGGAGAATCC & \\
\hline Abf-2-F & 176 & CCGTTCCCTITCCTTGCAC & (34) \\
\hline Abf-2-R & & GACGACCGCTTCGTTCTTG & \\
\hline Tir-1-F & 223 & TTGGGTGCACAAAGAGCTGA & (34) \\
\hline Tir-1-R & & GGTCGGTGTCGTTCTGTTCA & \\
\hline Nsy-1-F & 122 & AGCGGCTCGATCAACAAGAA & (34) \\
\hline Nsy-1-R & & CCCATTCCACCGATATGCGA & \\
\hline Sek-1-F & 158 & CACTGTITGGCGACGATGAG & (34) \\
\hline Sek-1-R & & ATTCCGTCCACGTTGCTGAT & \\
\hline Pmk-1-F & 115 & CCAAAAATGACTCGCCGTGA & (34) \\
\hline Pmk-1-R & & CTाTGCAGTTGGACGACGA & \\
\hline Bar-1-F & 119 & CATGGTAGTCCGCGACTTGT & (34) \\
\hline Bar-1-R & & CGAGAATTGACCAGCTCCAGA & \\
\hline Skn-1-F & 153 & CTGGCATCCTCTACCACCAC & (34) \\
\hline Skn-1-R & & TTGGTGATGATGGCCGTGTT & \\
\hline Dbl-1-F & 194 & TITGCGGCGAACAAATCGT & (34) \\
\hline Dbl-1-R & & TTCGCTGTTGCCTGTITGTG & \\
\hline Ced-3-F & 167 & AGAAGGAGCTTGCTAGAGAGGA & This study \\
\hline Ced-3-R & & ACTGCTITCACGATCTCCCG & \\
\hline Sod-3-F & 88 & GAAGATCGCCACCTGTGCAA & This study \\
\hline Sod-3-R & & CAAGTAGTAGGCGTGCTCCC & \\
\hline Ced-9-F & 146 & GTCTAATCTCGTTCGGCGGT & This study \\
\hline Ced-9-R & & CCAGCTCCGATTGTGTTCCT & \\
\hline Gapdh-F & 158 & ACTCGACCCACGGTCAATTC & (21) \\
\hline Gapdh-R & & ACTCGACAACGAAATCGGCT & \\
\hline
\end{tabular}

*All the PCR products amplified with the pairs of primers designed in this study have been verified by DNA sequencing.

\section{Response of the WT C. elegans in Gene Expression to DT104 Infection With or Without LB1 Pretreatment}

To examine the host immune response against $S$. typhimurium DT104 infection, the WT worms on day 2 of the life-span assay were selected based on the observation that the numbers of viable worms started to decrease on days 3-5 (Figure 1). The major 


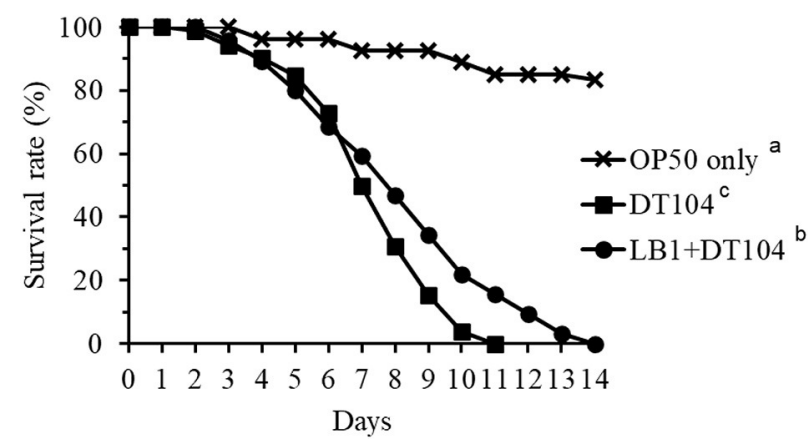

FIGURE 1 | Effect of pretreatment with isolate Lactobacillus zeae LB1 on the survival of Caenorhabditis elegans infected with Salmonella typhimurium DT104. The worms were first fed either Escherichia coli OP50 or LB1 at $10^{8}$ $\mathrm{CFU} / \mathrm{mL}$ for $18 \mathrm{~h}$ and then DT104 for the remaining days. Treatments: $\mathbf{\square}, E$. coli OP50 and then DT104; •, LB1 and then DT104; × , E. coli OP50 only. All the groups showing different letters were significant different $(P \leq 0.05)$ in their survival curves.

components in the p38 MAPK (tir-1, nsy-1, sek-1, and pmk-1), DAF/IGF (daf-16 and age-1), PCD (ced-3 and ced-9) pathways, previously identified antimicrobial peptides (lys-7, spp-1, abf-2, clec-85, clec-60, and $a b f-3$ ), and other reported defense molecules (sod-3, dbl-1, skn-1, and bar-1) were used as indicators for host response at the level of signaling transduction.

As shown in Figure 2, while the gene expression of $n s y-1$, age-1, abf-2, clec-85, and $d b l-1$ in the nematode infected with DT104 showed no significant changes $(P>0.05)$, the expression of daf-16, ced-3, ced-9, abf-3, lys-7, spp-1, and bar-1 genes was upregulated $(P \leq 0.05)$ compared with uninfected worms. In contrast, the expression of most selected genes associated with the p38 MAPK pathway (tir-1, sek-1, and pmk-1), clec-60 for antimicrobial peptide production, and $s k n-1$ and sod-3 for other defense molecules was decreased $(P \leq 0.05)$. Compared with the nematode infected with DT104 only, pretreatment of the worms with $L$. zeae LB1 significantly upregulated $(P \leq 0.05)$ the expression of almost all the selected genes except for age-1, ced-3, ced-9, abf-2, and dbl-1.

\section{Involvement of Cell Signaling and Production of Antimicrobial Peptides and Other Defense Molecules in the C. elegans Resistance to DT104 Infection}

To determine how cell signaling and production of antimicrobial peptides and other defense molecules affect the resistance of $C$. elegans to DT104 infection, the life-span of 14 different mutants infected with DT104 was investigated in comparison with the WT. The mutants included ag3 (defective in nsy-1), ag1 (sek1), and $\mathrm{km} 25$ (pmk-1) defective in the p38 MAPK pathway; mu86 (daf-16) and hx546 (age-1) defective in the DAF/IGF pathway; mutants n717 (ced-3), n1950 (ced-9), and the double mutant n2812/n717 (ced-9; ced-3) defective in the PCD pathway; ok3366 (abf-3), ok1384 (lys-7), and ok2703 (spp-1) defective in antimicrobial peptide production; and nk3 (dbl-1), gk235 (sod-3), and zu67 (skn-1) defective in the production of other molecules with a defense function.

The results showed that the life-span of the tested mutants ag3, ag1, and km25 defective in the p38 MAPK pathway was all significantly shorter than that of the WT nematode when exposed to DT104 (Figure 3A). Mutants n717, n1950, and n2812/n717 defective in the PCD pathway became more susceptible to DT104 infection (Figure 3C). The life-span of these mutants with one gene mutation was reduced by more than $30 \%$ compared to the WT. Furthermore, the mutant (n2812/n717) with two mutations (defective in both ced-3 and ced-9) had only a half of life-span of the WT. For the remaining mutants, mutants mu86 (defective in daf-16), ok3366 (defective in abf-3), ok1384 (defective in lys7 ), and zu67 (defective in $s k n-1)$ showed a shorter life-span $(P$ $\leq 0.05$ ) than the WT (Figures 3B,D,E). In contrast, the lifespan of the mutants that are defective in age-1 (hx546), spp-1 (ok2703), or $d b l-1$ (nk3) were more resistant to DT104 infection with an increased life-span compare with the WT nematode (Figures 3B,D,E). Mutant gk235 (defective in sod-3) was an exception, which had a similar life-span of the WT when exposed to DT104 (Figure 3E).

\section{Regulation of Cell Signaling and Production of Antimicrobial Peptides in C. elegans by LB1}

To determine the role of $L$. zeae LB1 in regulating the cell signaling of $C$. elegans to resist $S$. typhimurium DT104 infection, 7 mutants were examined for their resistance to DT104 infection after pretreatment with LB1. Interestingly, only the three mutants that are defective in $n s y-1$, sek-1, or $p m k-1$ demonstrated no changes to DT104 infection even though they were pretreated with LB1 (Figures 4A-C), suggesting no protection from the isolate. These three mutants were shown to be more susceptible to DT104 infection than the WT in the life-span assay without LB1 pretreatment (Figure 3A). The mutants that are defective in daf-16, ced-3, or ced-9 and were also shown to be more susceptible to DT104 infection than the WT in the previous life-span assay without LB1 pretreatment (Figures 3B,C), however, the mutants exhibited a significantly increased life-span $(P \leq 0.05)$ after the pretreatment with LB1 (Figures 4D,F,G). The pretreatment with LB1 also significantly increased $(P \leq 0.05)$ the life-span of the mutant defective in age-1 (Figure 4E) that was more resistant to DT104 infection compared to the WT in the previous assay without LB1 pretreatment (Figure 3B).

To determine if $L$. zeae LB1 regulates the production of antimicrobial peptides and other defense molecules in $C$. elegans responding to DT104 infection, the life-span of mutants ok3366 (defective in $a b f-3$ ), ok1384 (defective in lys-7), ok2703 (defective in $s p p-1$ ), zu67 (defective in $s k n-1$ ), gk235 (defective in sod-3), and nk3 (defective in $d b l-1$ ) were investigated with or without LB1 pretreatment. As shown in Figure 5, both mutants defective in $a b f-3$ or lys-7 had no changes in the lifespan regardless of LB1 pretreatment, suggesting no protection from LB1 (Figures 5A,B). However, pretreatment with LB1 significantly increased $(P \leq 0.05)$ the life-span of the mutants that are defective in sod-3, skn-1, or $d b l-1$ (Figures 5D-F) and showed 


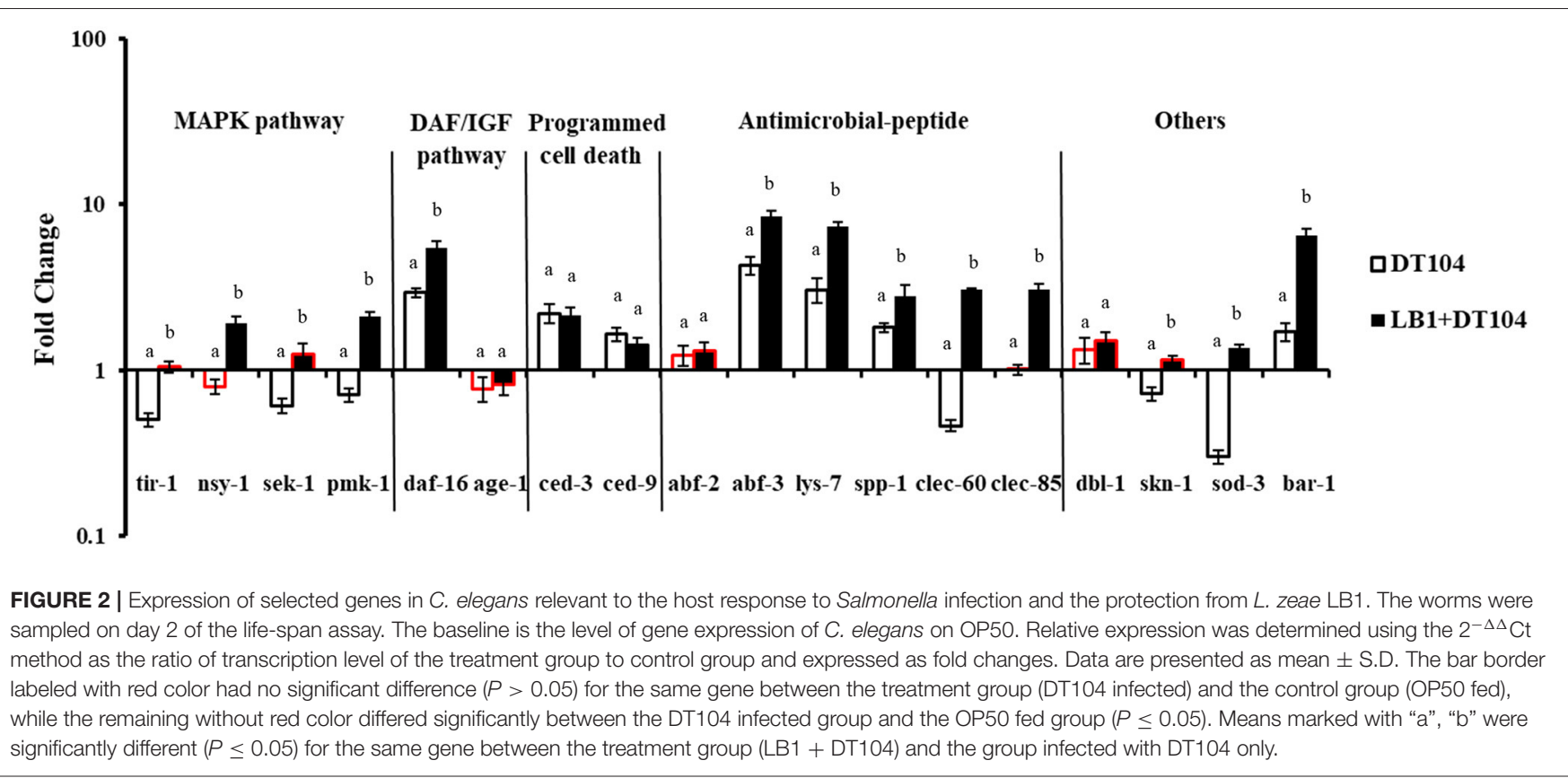

A

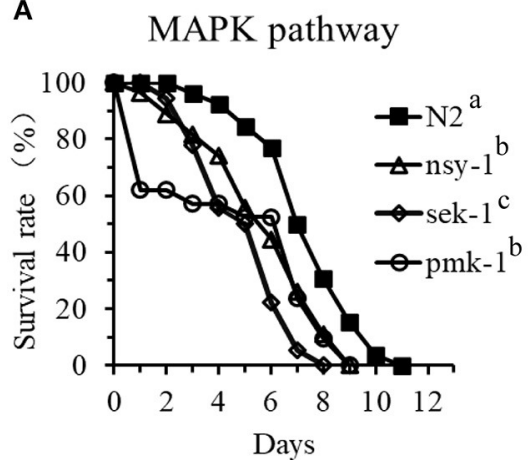

D

antimicrobial-peptide

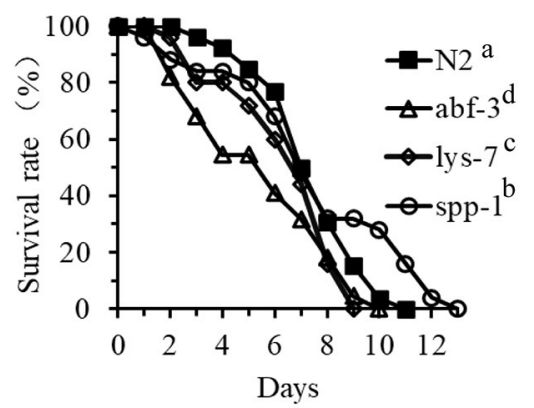

B

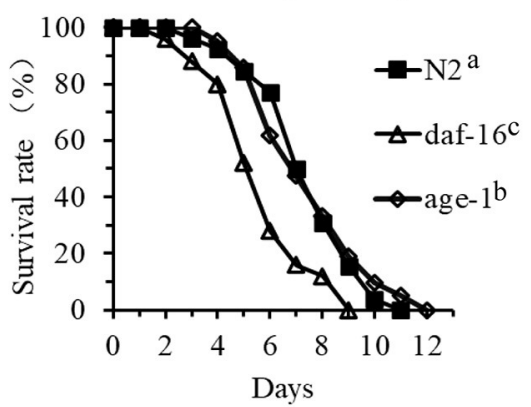

E

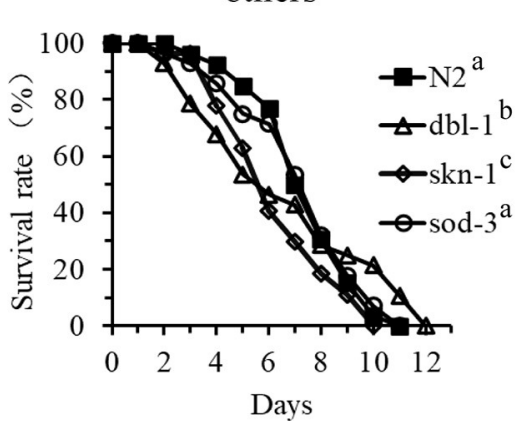

C Programmed cell death

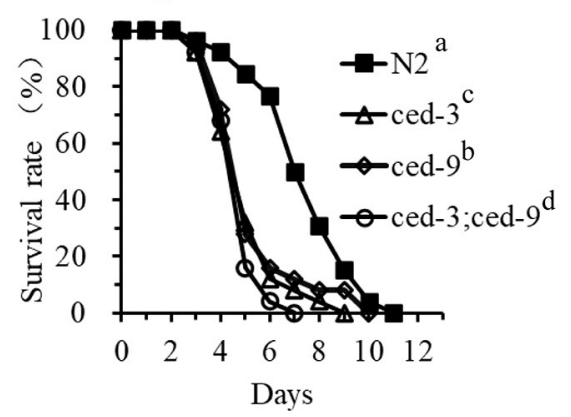

FIGURE 3 | The life-span assay of C. elegans mutants in comparison with the WT that are infected with S. typhimurium DT104. (A) Survival curves of p38-MAPK pathway mutants: nsy-1(ag3), sek-1(ag1), pmk-1(km25); (B) Survival curves of DAF/IGF pathway mutants: daf-16(mu86), age-1(hx546); (C) Survival curves of PCD pathway mutants: ced-9(n1950), ced-3(n717), or ced-9;ced-3(n1950;n717); (D) Survival curves of the mutants defective in antimicrobial peptide genes:

abf-3(ok3366), lys-7(ok1384), or spp-1(ok2703); (E) Survival curves of other mutants with a defense function: $d b /$-1(nk3), skn-1(zu67), sod-3(gk235). All treatments were feed with salmonella DT104 as food instead of $E$. coli OP50. All the groups showing different letters were significant different $(P \leq 0.05)$ in their survival curves. 


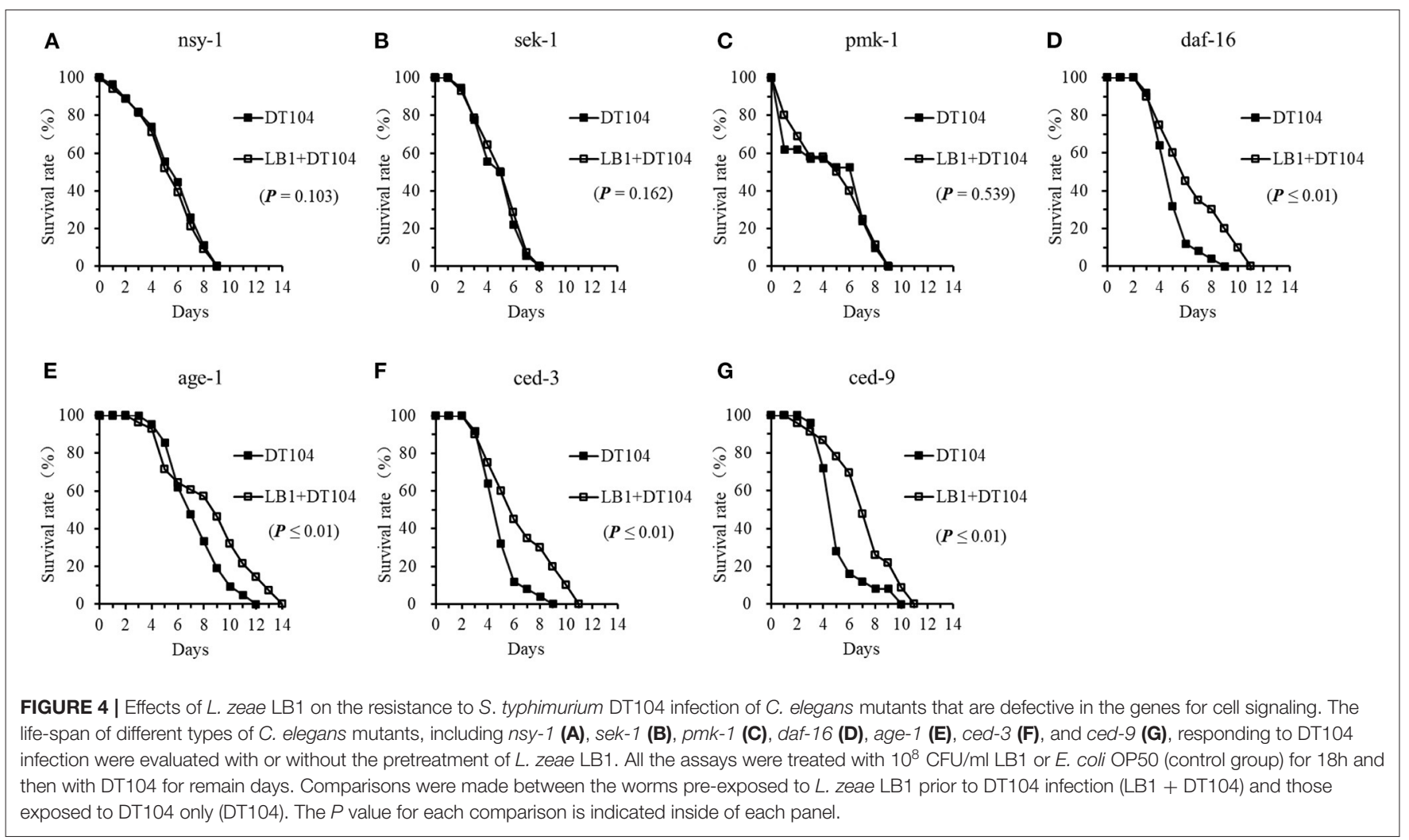

no change, decreased or increased resistance to DT104 infection in the previous assay without LB1 pretreatment, respectively (Figure 3E). In contrast to these observations, the mutant defective in $s p p-1$ became more susceptible to DT104 infection after LB1 pretreatment (Figure 5C), which was more resistant to the infection without LB1 pretreatment (Figure 3D). In all the life-span assays with the mutants, the WT behaved similarly as in the previous assays with or without LB1 pretreatment.

\section{DISCUSSION}

The suitability of $C$. elegans for the studies of host immune response to bacteria including both foodborne pathogens and probiotics was further demonstrated recently $(14,34,36-38)$. The nematode innate immune system is highly conserved and can be quickly activated through several immune regulatory pathways to protect the host against bacterial infection (7). Among them, the $\mathrm{p} 38 \mathrm{MAPK}$ and DAF/IGF signaling pathways play a vital role in combating bacterial infection in the intestine. For example, these two pathways were shown to be important in controlling Salmonella infection $(10,20)$. In addition, the PCD pathway was also found to have a role in the host resistance to Salmonella infection (23). Interestingly, we recently identified the positive role of both serotonin and dopamine in the host defense of $C$. elegans to S. typhimurium infection through regulation of the p38 MAPK and DAF/IGF pathways (21). To further elucidate this regulation, the present study has investigated the role of particular members in those pathways and provided new insight into the regulation of both cell signaling and production of antimicrobial peptides and other defense molecules in C. elegans.

In the present study, four genes (tir-1,nsy-1, sek-1, and pmk1) in the p38 MAPK pathway, two genes (daf-16 and age-1) in the DAF/IGF pathway, two genes (ced-3, ced-9) in the PCD pathway, six genes $(a b f-2, a b f-3, l y s-7, s p p-1$, clec-60, and clec$85)$ encoding antimicrobial peptides, and four other genes ( $d b l$ 1, skn-1, sod-3, and bar-1) reported previously with a defense function $(26,39,40)$ were initially investigated for the possible involvement in the immune response of the WT nematode by examining their expression. When infected with S. typhimurium DT104, the expression of daf-16, ced-3, ced-9, abf-3, lys-7, spp1 , and bar-1 genes was upregulated $(P \leq 0.05)$, whereas others such as clec-60, skn-1, and sod-3 were suppressed. In particular, the expression of all the selected genes associated with the p38 MAPK pathway except for $n s y-1$ was downregulated $(P \leq 0.05)$. These results suggested that the p38 MAPK pathway was one of the major targets by DT104 while the PCD and DAF/IGF pathways were also affected. The results from the life-span assay with various mutants supported the notion. The mutants that are defective in $n s y-1$, sek-1, or pmk-1 (the major components in the p38 MAPK pathway), in ced-3 or ced-9 (the major components of PCD pathway), or in daf-16 (a major component of DAF/IGF pathway) all became more susceptible to DT104 infection with over $20-30 \%$ reduction in the life-span compared to the WT (Figures 3A-C). Furthermore, the life-span of the double mutant defective in both ced-3 and ced-9 had only half of the lifespan of the WT. Based on the data described above, it appears that the response of C. elegans to DT104 infection is mediated 


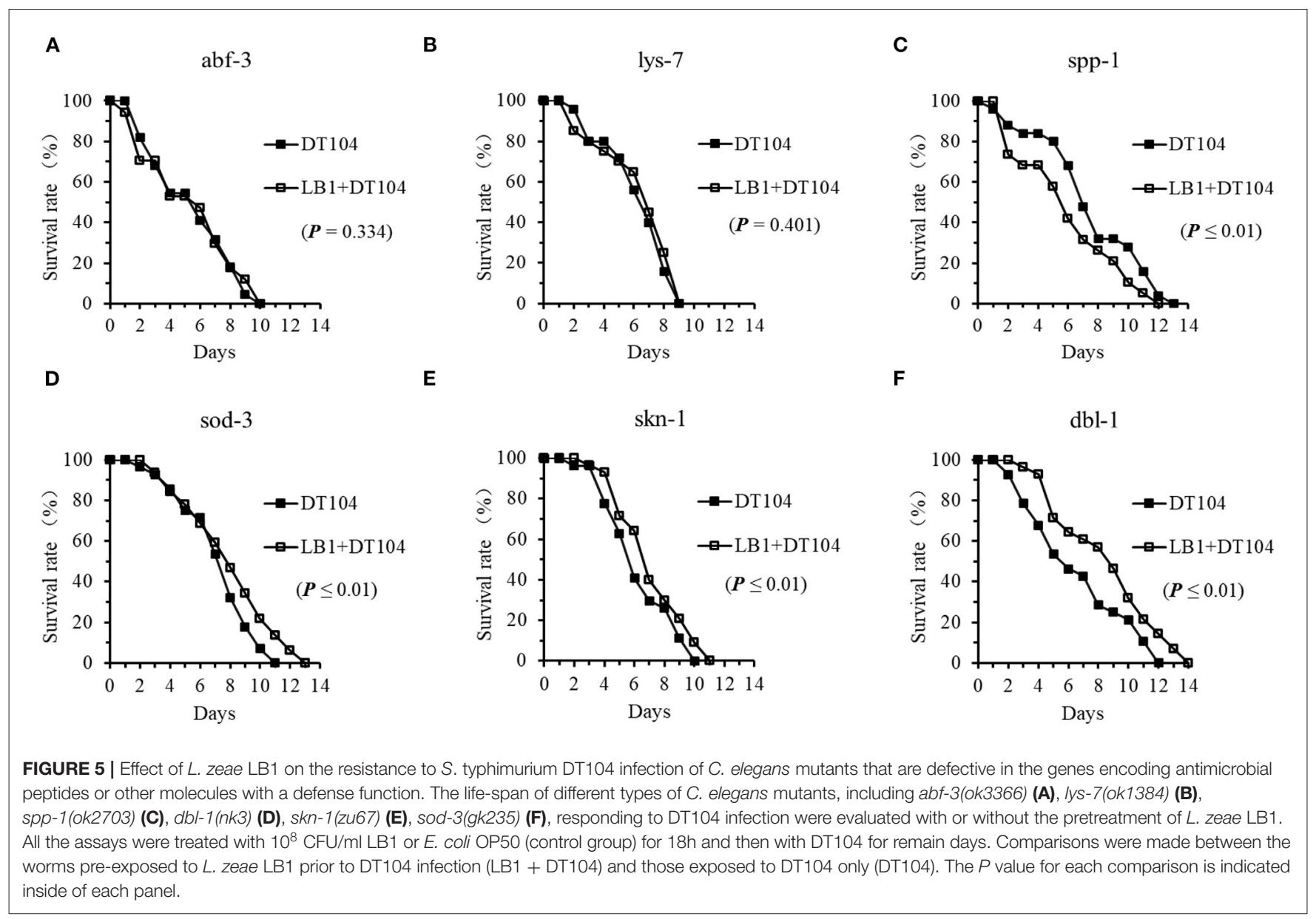

mainly through the regulation of the p38 MAPK, DAF/IGF, and PCD pathways.

Although many important innate immune pathways and effectors have been identified in C. elegans, there are differences in the host responses to different bacterial pathogens especially in the regulation of antimicrobial peptide production $(28,29$, $31,41)$. Analyses of mutations in different genes associated with the responses have identified downstream proteins involved in pathogen defense, such as LYS (lysozyme) family, ABF (Ascaris suum antibacterial factor) family, SPP (Caenopores are the saposin-like proteins) family, and C-type lectins family $(29,30)$. However, the role of these antimicrobial peptides in response to bacterial infection is yet to be fully elucidated $(7,14,42)$. In the present study, the transcription of genes that encode for antimicrobial peptides including $a b f-3$, lys-7, and $s p p-1$ was all significantly upregulated in the WT nematode when it was exposed to DT104. This suggests a vital role for antimicrobial peptides in the defense of C. elegans against Salmonella infection. This notion is also supported by the fact that $C$. elegans with a mutation in an antimicrobial peptide gene (lys-7, or abf-3) showed significant shorter life-span than the WT when the mutants were subjected to DT104 infection (Figure 3D). In our previous report, the mutant defective in $s p p-1$ showed shorter life-span than the WT when the nematode was subjected to ETEC infection only, but no changes in the life-span regardless of the pretreatment with L. zeae LB1 (34). However, in the current study the same mutant had significantly longer life-span than the WT when exposed to S. typhimurium DT104 only (Figure 3D), but a shorter life-span than the WT after the pretreatment with L. zeae LB1 (Figure 5C). While the reason underlying these observations is unclear, it provides another piece of evidence that the role of antimicrobial peptides can vary in the host response to different pathogens.

There were several reports recently that Lactobacillus can confer health benefits to C. elegans including life-span extending, protection against pathogen infection, and prevention from abiotic stress (20,43-45). Park et al. (38) reported that probiotic L. fermentum strain JDFM216 stimulated the longevity and immune response of $C$. elegans through a nuclear hormone receptor (NHR) family and PMK-1 signaling (38). Pediococcus acidilactici P25 strain affected expression of the genes related to innate immune response and upregulated the abundance of transcripts in multiple pathways of C. elegans, including peroxisome, longevity, and MAPK pathways (42). Nevertheless, these reports have not yet identified immunomodulatory effects of Lactobacillus on the targeting sites downstream of the p38 MAPK and DAF/IGF pathways. Our previous study found that a selected strain L. zeae LB1 could provide protection 


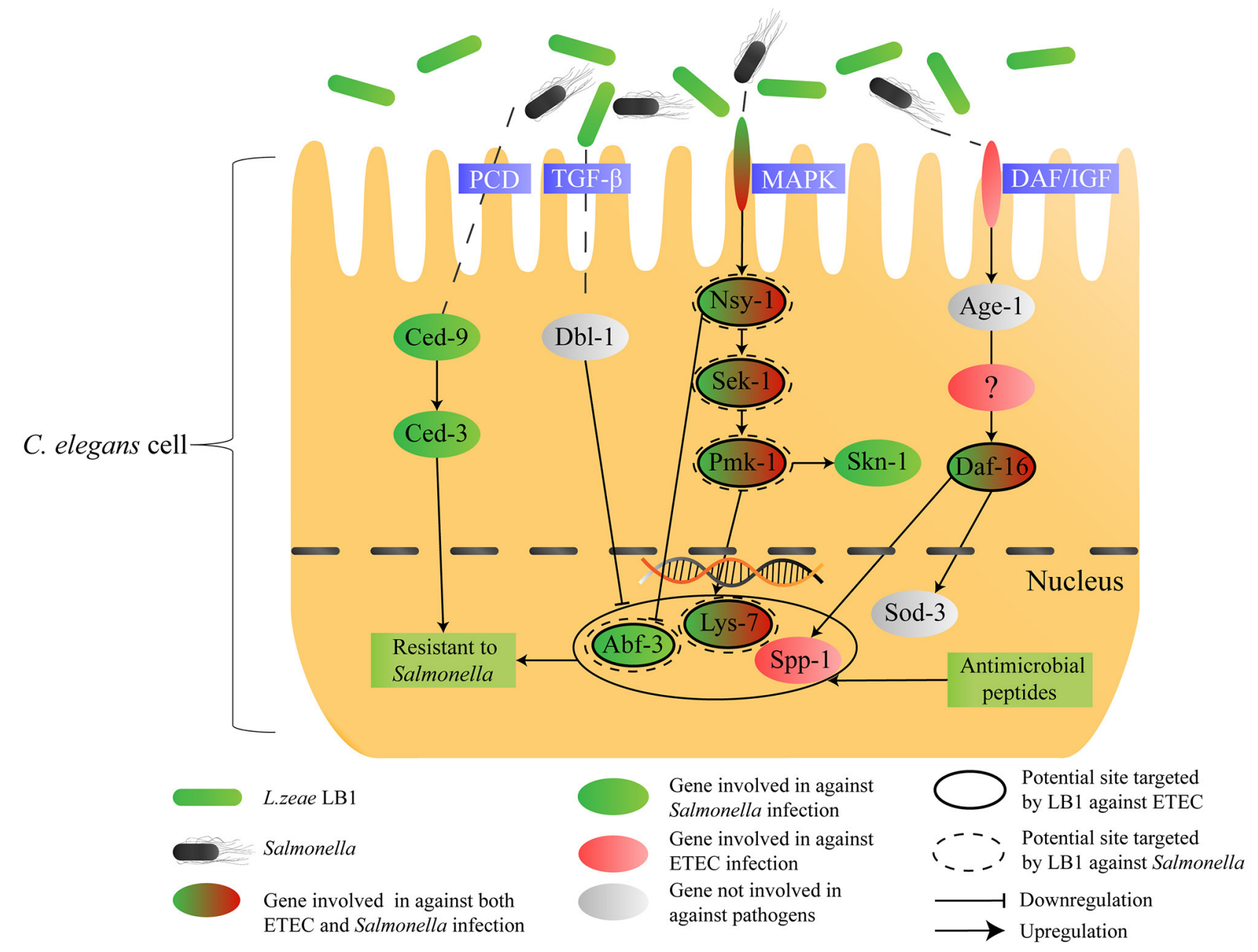

FIGURE 6 | Schematic diagram speculating the immunomodulatory mechanism by L. zeae. The hypothesis was based on the data from both life-span assays and gene expression experiments of various $C$. elegans mutants either pretreated or not with L. zeae LB1 before S. typhimurium DT104 infection in the present study and on the data published previously on the regulation of $C$. elegans cell signaling by L. zeae LB1 against ETEC infection (34).

by regulating $C$. elegans cell signaling through the p38MAPK and DAF/IGF pathways to control the production of antimicrobial peptides and defense molecules (34). The same strain was also investigated for its immune-regulatory effects against S. typhimurium DT104 infection in the present study. The data from both the gene expression and life-span studies indicate that the protection offered by L. zeae LB1 against Salmonella-caused death also involved the p38 MAPK, DAF/IGF, and PCD pathways as well as the production of antimicrobial peptides and other defense molecules in C. elegans. In particular, five genes including $n s y-1$, sek-1, pmk-1, abf3 , and $l y s-7$ in the p38 MAPK pathway or for antimicrobial peptide production appear to be the sites regulated by LB1 as LB1 pretreatment provided no protection to the corresponding mutants (Figures $\mathbf{4 A - C}, \mathbf{5 A}, \mathbf{B}$ ). Kim et al. (20) reported that L. acidophilus NCFM activates the p38 MAPK pathway (via TIR-1 and PMK-1) and the $\beta$-catenin signaling pathway (via BAR-1) against Gram-positive bacteria while it has no effect on Gram-negative ( $P$. aeruginosa or S. typhimurium) (20). Very recently, we demonstrated that the protection of $L$. zeae LB1 against S. typhimurium DT104 infection was mediated by dopamine through both the p38 MAPK and DAF/IGF pathways (21). These observations with the data described in the present study suggest that the protection offered by Lactobacillus can be species or even strain-specific with different immunomodulation mechanisms.

In conclusion, the present study has revealed: [1] the host response of $C$. elegans to $S$. typhimurium DT104 infection mainly involves the $\mathrm{p} 38 \mathrm{MAPK}, \mathrm{DAF} / \mathrm{IGF}$ PCD pathways and production of antimicrobial peptides and defense molecules; [2] Nsy-1, Sek-1, and Pmk-1 (MAPK pathway) as well as Lys-7 and Abf-3 (antimicrobial peptides) appear to be the sites regulated by L. zeae LB1, leading to the protection; [3] L. zeae LB1 can induce different immune responses in $C$. elegans when the nematode is infected by different pathogens. To summarize the findings from the present study, a schematic diagram has been generated 
(Figure 6), which also proposes a possible immunomodulatory mechanism by L. zeae.

\section{DATA AVAILABILITY STATEMENT}

The original contributions presented in the study are included in the article/supplementary material, further inquiries can be directed to the corresponding author/s.

\section{AUTHOR CONTRIBUTIONS}

$\mathrm{MZ}$ and XL performed the experiments. MZ, HY, XL, and JG analyzed data. MZ and JG wrote the manuscript. MZ, HY, and JG designed the experiments. JG conceived the research. All authors approved the final version of manuscript.

\section{REFERENCES}

1. Matsuzaki T. Modulating immune responses with probiotic bacteria. Immunol Cell Biol. (2000) 78:67-73. doi: 10.1046/j.1440-1711.2000.00887.x

2. Lebeer S, Vanderleyden J, De Keersmaecker SC. Host interactions of probiotic bacterial surface molecules: comparison with commensals and pathogens. Nat Rev Microbiol. (2010) 8:171-84. doi: 10.1038/nrmicro2297

3. Signorini ML, Soto LP, Zbrun MV, Sequeira GJ, Rosmini MR, Frizzo LS. Impact of probiotic administration on the health and fecal microbiota of young calves: a meta-analysis of randomized controlled trials of lactic acid bacteria. Res Vet Sci. (2012) 93:250-8. doi: 10.1016/j.rvsc.2011.05.001

4. Brestoff JR, Artis D. Commensal bacteria at the interface of host metabolism and the immune system. Nat Immunol. (2013) 14:676. doi: 10.1038/ni.2640

5. Chambers JR, Gong J. The intestinal microbiota and its modulation for Salmonella control in chickens. Food Res Int. (2011) 44:3149-59. doi: 10.1016/j.foodres.2011.08.017

6. Rodriguez-Sorrento A, Castillejos L, Lopez-Colom P, Cifuentes-Orjuela G, Rodriguez-Palmero M, Moreno-Munoz JA, et al. Effects of Bifidobacterium longum Subsp. infantis CECT 7210 and Lactobacillus rhamnosus HN001, combined or not with oligofructose-enriched inulin, on weaned pigs orally challenged with Salmonella Typhimurium. Front Microbiol. (2020) 11:2012. doi: $10.3389 /$ fmicb.2020.02012

7. Zyl WFV, Deane SM, Dicks LMT. Molecular insights into probiotic mechanisms of action employed against intestinal pathogenic bacteria. Gut Microbes. (2020) 12:25. doi: 10.1080/19490976.2020.1831339

8. Ikeda $\mathrm{T}$, Yasui $\mathrm{C}$, Hoshino $\mathrm{K}$, Arikawa $\mathrm{K}$, Nishikawa $\mathrm{Y}$. Influence of lactic acid bacteria on longevity of Caenorhabditis elegans and host defense against Salmonella enterica serovar Enteritidis. Appl Environ Microbiol. (2007) 73:6404-9. doi: 10.1128/AEM.00704-07

9. Wang C, Wang J, Gong J, Yu H, Pacan JC, Niu Z, et al. Use of Caenorhabditis elegans for preselecting lactobacillus isolates to control Salmonella typhimurium. J Food Protect. (2011) 74:86-93. doi: 10.4315/0362-028X.JFP-10-155

10. Lee J, Choe J, Kim J, Oh S, Park S, Kim S, et al. Heat-killed Lactobacillus spp. cells enhance survivals of Caenorhabditis elegans against Salmonella and Yersinia infections. Lett Appl Microbiol. (2015) 61:523-30. doi: 10.1111/lam.12478

11. Tan M-W, Mahajan-Miklos S, Ausubel FM. Killing of Caenorhabditis elegans by Pseudomonas aeruginosa used to model mammalian bacterial pathogenesis. Proc Natl Acad Sci USA. (1999) 96:715-20. doi: 10.1073/pnas.96.2.715

12. Garsin DA, Sifri CD, Mylonakis E, Qin X, Singh KV, Murray BE, et al. A simple model host for identifying Gram-positive virulence factors. Proc Natl Acad Sci USA. (2001) 98:10892-7. doi: 10.1073/pnas.191378698

13. Kwok TC, Ricker N, Fraser R, Chan AW, Burns A, Stanley EF, et al. A smallmolecule screen in C. elegans yields a new calcium channel antagonist. Nature. (2006) 441:91-5. doi: 10.1038/nature04657

\section{FUNDING}

This work was supported by Agriculture \& Agri-Food Canada (AAFC) and Swine Innovation Porc through the Swine Science Cluster Program (AAFC Project\# J-002109) and the National Natural Science Foundation of China (No: 31601455).

\section{ACKNOWLEDGMENTS}

$\mathrm{MZ}$ and $\mathrm{XL}$ were visiting graduate students to AAFC, financially supported by the China Scholarship Council through the MOEAAFC Ph.D. Research Program. We thank Dr. Jason Carere in the Food Research Division of Health Canada for reviewing this manuscript.

14. Kumar A, Baruah A, Tomioka M, Iino Y, Kalita MC, Khan M. Caenorhabditis elegans: a model to understand host-microbe interactions. Cell Mol Life Sci. (2020) 77:1229-49. doi: 10.1007/s00018-019-03319-7

15. Aballay A, Yorgey P, Ausubel FM. Salmonella typhimurium proliferates and establishes a persistent infection in the intestine of Caenorhabditis elegans. Curr Biol. (2000) 10:1539-42. doi: 10.1016/S0960-9822(00)00830-7

16. Labrousse A, Chauvet S, Couillault C, Léopold Kurz C, Ewbank JJ. Caenorhabditis elegans is a model host for Salmonella typhimurium. Curr Biol. (2000) 10:1543-5. doi: 10.1016/S0960-9822(00)00833-2

17. Sifri CD, Begun J, Ausubel FM, Calderwood SB. Caenorhabditis elegans as a model host for Staphylococcus aureus pathogenesis. Infect Immunity. (2003) 71:2208-17. doi: 10.1128/IAI.71.4.2208-2217.2003

18. Tenor JL, Mccormick BA, Ausubel FM, Aballay A. Caenorhabditis elegans-based screen identifies salmonella virulence factors required for conserved host-pathogen. Interactions. (2004) 14:1018-24. doi: 10.1016/j.cub.2004.05.050

19. Zhou M, Yu H, Yin X, Sabour PM, Chen W, Gong J. Lactobacillus zeae protects Caenorhabditis elegans from enterotoxigenic escherichia coli-caused death by inhibiting enterotoxin gene expression of the pathogen. PLoS ONE. (2014) 9:e89004. doi: 10.1371/journal.pone.0089004

20. Kim Y, Mylonakis E. Caenorhabditis elegans immune conditioning with the probiotic bacterium Lactobacillus acidophilus strain NCFM enhances gram-positive immune responses. Infect Immunity. (2012) 80:2500-8. doi: 10.1128/IAI.06350-11

21. Liu X, Jiang L, Li L, Yu H, Nie S, Xie M, et al. The role of neurotransmitters in the protection of Caenorhabditis elegans for Salmonella infection by Lactobacillus. Front Cell Infect Microbiol. (2020) 10:554052. doi: $10.3389 /$ fcimb.2020.554052

22. Yang X, Brisbin J, Yu H, Wang Q, Yin F, Zhang Y, et al. Selected lactic acid-producing bacterial isolates with the capacity to reduce Salmonella translocation and virulence gene expression in chickens. PLoS ONE. (2014) 9:e93022. doi: 10.1371/journal.pone.0093022

23. Aballay A, Ausubel FM. Programmed cell death mediated by ced-3 and ced-4 protects Caenorhabditis elegans from Salmonella typhimurium-mediated killing. Proc Natl Acad Sci USA. (2001) 98:2735-9. doi: $10.1073 /$ pnas.041613098

24. Kurz CL, Ewbank JJ. Caenorhabditis elegans: an emerging genetic model for the study of innate immunity. Nat Rev Genet. (2003) 4:380-90. doi: $10.1038 / \mathrm{nrg} 1067$

25. Murphy CT, Mccarroll SA, Bargmann CI, Fraser A, Kamath RS, Ahringer J, et al. Genes that act downstream of DAF-16 to influence the lifespan of Caenorhabditis elegans. Nature. (2003) 424:277. doi: 10.1038/nature01789

26. Zugasti O, Ewbank JJ. Neuroimmune regulation of antimicrobial peptide expression by a noncanonical TGF- $\beta$ signaling pathway in Caenorhabditis elegans epidermis. Nat Immunol. (2009) 10:249-56. doi: 10.1038/ ni. 1700 
27. Irazoqui JE, Urbach JM, Ausubel FM. Evolution of host innate defence: insights from Caenorhabditis elegans and primitive invertebrates. Nat Rev Immunol. (2010) 10:47-58. doi: 10.1038/nri2689

28. Mallo GV, Kurz CL, Couillault C, Pujol N, Granjeaud S, Kohara Y, et al. Inducible antibacterial defense system in C. elegans. Curr Biol. (2002) 12:120914. doi: 10.1016/S0960-9822(02)00928-4

29. Couillault C, Pujol N, Reboul J, Sabatier L, Guichou J-F, Kohara Y, et al. TLRindependent control of innate immunity in Caenorhabditis elegans by the TIR domain adaptor protein TIR-1, an ortholog of human SARM. Nat Immunol. (2004) 5:488-94. doi: 10.1038/ni1060

30. Bogaerts A, Beets I, Schoofs L, Verleyen P. Antimicrobial peptides in Caenorhabditis elegans. Invertebrate Survival J. (2010) 7:45-52.

31. O'rourke D, Baban D, Demidova M, Mott R, Hodgkin J.. Genomic clusters, putative pathogen recognition molecules, and antimicrobial genes are induced by infection of C. elegans with M. nematophilum. Genome Res. (2006) 16:100516. doi: 10.1101/gr.50823006

32. Breger J, Fuchs BB, Aperis G, Moy TI, Ausubel FM, Mylonakis E. Antifungal chemical compounds identified using a C. elegans pathogenicity assay. PLoS Pathogens. (2007) 3:e18. doi: 10.1371/journal.ppat.0030018

33. Poppe C, Smart N, Khakhria R, Johnson W, Prescott J. Salmonella typhimurium DT104: a virulent and drug-resistant pathogen. Can Vet J La Revue Vét Can. (1998) 39:559-65.

34. Zhou M, Liu X, Yu H, Yin X, Nie S-P, Xie M-Y, et al. Cell signaling of Caenorhabditis elegans in response to enterotoxigenic Escherichia coli infection and Lactobacillus zeae protection. Front Immunol. (2018) 9:1745. doi: $10.3389 /$ fimmu.2018.01745

35. Livak KJ, Schmittgen TD. (2001). Analysis of Relative Gene Expression Data Using Real-Time Quantitative PCR and the 2(-Delta Delta C (T)) Method. Methods. 25, 402-408. doi: 10.1006/meth.2001.1262

36. Curt A, Zhang J, Minnerly J, Jia K. Intestinal autophagy activity is essential for host defense against Salmonella typhimurium infection in Caenorhabditis elegans. Dev Comp Immunol. (2014) 45:214-8. doi: 10.1016/j.dci.2014.03.009

37. Prithika U, Deepa V, Balamurugan K. External induction of heat shock stimulates the immune response and longevity of Caenorhabditis elegans towards pathogen exposure. Innate Immun. (2016) 22:466-78. doi: $10.1177 / 1753425916654557$

38. Park MR, Ryu S, Maburutse BE, Oh NS, Kim SH, Oh S, et al. Probiotic Lactobacillus fermentum strain JDFM216 stimulates the longevity and immune response of Caenorhabditis elegans through a nuclear hormone receptor. Sci Rep. (2018) 8:7441. doi: 10.1038/s41598-01825333-8
39. Keshet A, Mertenskötter A, Winter SA, Brinkmann V, Dölling R, Paul RJ. PMK-1 p38 MAPK promotes cadmium stress resistance, the expression of SKN-1/Nrf and DAF-16 target genes, and protein biosynthesis in Caenorhabditis elegans. Mol Genet Genom Mgg. 292:1341-61. doi: 10.1007/s00438-017-1351-Z

40. Liang Z, Yang Z, Liu R, Zheng X, Min Z, Guo H, et al. The transcription factor DAF-16 is essential for increased longevity in $C$. elegans exposed to Bifidobacterium longum BB68. Sci Rep. (2017) 7:7408. doi: 10.1038/s41598-017-07974-3

41. Alper S, Mcbride SJ, Lackford B, Freedman JH, Schwartz DA. Specificity and complexity of the Caenorhabditis elegans innate immune response. Mol Cell Biol. (2007) 27:5544-53. doi: 10.1128/MCB.02070-06

42. Tan K, Deng D, Ma X, Cui Y, Tian Z. Pediococcus acidilactici P25 protected Caenorhabditis elegans against enterotoxigenic Escherichia coli K88 infection and transcriptomic analysis of its potential mechanisms. BioMed Res Int. (2020) 2020:7340312. doi: 10.1155/2020/7340312

43. Grompone G, Martorell P, Llopis S, Gonzalez N, Genoves S, Paula Mulet A, et al. Anti-inflammatory Lactobacillus rhamnosus CNCM I-3690 strain protects against oxidative stress and increases lifespan in Caenorhabditis elegans. PLoS ONE. (2012) 7:52493. doi: 10.1371/journal.pone.0052493

44. Zhao Y, Yu X, Jia R, Yang R, Rui Q, Wang D. Lactic acid bacteria protects Caenorhabditis elegans from toxicity of graphene oxide by maintaining normal intestinal permeability under different genetic backgrounds. Sci Rep. (2015) 5:17233. doi: $10.1038 /$ srep 17233

45. Kamaladevi A, Balamurugan K. Lactobacillus casei triggers a TLR mediated RACK-1 dependent p38 MAPK pathway in Caenorhabditis elegans to resist Klebsiella pneumoniae infection. Food Funct. (2016) 7:3211-23. doi: 10.1039/C6FO00510A

Conflict of Interest: The authors declare that the research was conducted in the absence of any commercial or financial relationships that could be construed as a potential conflict of interest.

Copyright (c) 2021 Her Majesty the Queen in Right of Canada, as represented by the Minister of Agriculture and Agri-Food Canada. This is an open-access article distributed under the terms of the Creative Commons Attribution License (CC $B Y)$. The use, distribution or reproduction in other forums is permitted, provided the original author(s) and the copyright owner are credited and that the original publication in this journal is cited, in accordance with accepted academic practice. No use, distribution or reproduction is permitted which does not comply with these terms. 